\title{
Realization of the Hofstadter Hamiltonian with Ultracold Atoms in Optical Lattices
}

\author{
M. Aidelsburger, ${ }^{1,2}$ M. Atala, ${ }^{1,2}$ M. Lohse, ${ }^{1,2}$ J. T. Barreiro, ${ }^{1,2}$ B. Paredes, ${ }^{3}$ and I. Bloch ${ }^{1,2}$ \\ ${ }^{1}$ Fakultät für Physik, Ludwig-Maximilians-Universität, Schellingstrasse 4, 80799 München, Germany \\ ${ }^{2}$ Max-Planck-Institut für Quantenoptik, Hans-Kopfermann-Strasse 1, 85748 Garching, Germany \\ ${ }^{3}$ Instituto de Física Teórica CSIC/UAM C /Nicolás Cabrera, 13-15 Cantoblanco, 28049 Madrid, Spain
}

(Received 1 August 2013; published 28 October 2013)

\begin{abstract}
We demonstrate the experimental implementation of an optical lattice that allows for the generation of large homogeneous and tunable artificial magnetic fields with ultracold atoms. Using laser-assisted tunneling in a tilted optical potential, we engineer spatially dependent complex tunneling amplitudes. Thereby, atoms hopping in the lattice accumulate a phase shift equivalent to the Aharonov-Bohm phase of charged particles in a magnetic field. We determine the local distribution of fluxes through the observation of cyclotron orbits of the atoms on lattice plaquettes, showing that the system is described by the Hofstadter model. Furthermore, we show that for two atomic spin states with opposite magnetic moments, our system naturally realizes the time-reversal-symmetric Hamiltonian underlying the quantum spin Hall effect; i.e., two different spin components experience opposite directions of the magnetic field.
\end{abstract}

DOI: 10.1103/PhysRevLett.111.185301

Ultracold atoms in optical lattices constitute a unique experimental setting to study condensed matter Hamiltonians in a clean and well-controlled environment [1], even in regimes not accessible to typical condensed matter systems [2]. Especially intriguing is their promising potential to realize and probe topological phases of matter, for example, by utilizing the newly developed quantum optical high-resolution detection and manipulation techniques $[3,4]$. One compelling possibility in this direction is the quantum simulation of electrons moving in a periodic potential exposed to a large magnetic field, described by the Hofstadter-Harper Hamiltonian [5,6]. For a filled band of fermions, this model realizes the paradigmatic example of a topological insulator that breaks time-reversal symmetry-the quantum Hall insulator. Moreover, the atomic realization of time-reversal-symmetric topological insulators based on the quantum spin Hall effect [7] promises new insights for spintronic applications.

The direct quantum simulation of orbital magnetism in ultracold quantum gases is, however, hindered by the charge neutrality of atoms, which prevents them from experiencing a Lorentz force. Overcoming this limitation through the engineering of synthetic gauge potentials is currently a major topic in cold-atom research. Artificial magnetic fields were first accomplished using the Coriolis force in a rotating atomic gas $[8,9]$ and later by inducing Berry's phases through the application of Raman lasers [10,11]. Recently, staggered magnetic fields in optical lattices were achieved using laser-induced tunneling in superlattice potentials [12] or through dynamical shaking [13]. In one dimension, tunable gauge fields have been implemented in an effective "Zeeman lattice" [14] and using periodic driving [15]. Furthermore, the free-space spin Hall effect was observed using Raman dressing [16]. Despite intense research efforts,
PACS numbers: 67.85.- d, 03.65.Vf, 03.75.Lm, 73.20.-r

2D optical lattices featuring topological many-body phases have so far been beyond the reach of experiments.

In this Letter, we demonstrate the first experimental realization of an optical lattice that allows for the generation of large tunable homogeneous artificial magnetic fields. The technique is based on our previous work on staggered magnetic fields [12]. The main idea is closely related to early proposals by Jaksch and Zoller [17] and subsequent work $[18,19]$. However, it does not rely on the internal structure of the atom, which makes it applicable to a larger variety of atomic species, including fermionic atoms like ${ }^{6} \mathrm{Li}$ and ${ }^{40} \mathrm{~K}$. We use laser-assisted tunneling in a tilted optical lattice through periodic driving with a pair of far-detuned running-wave beams [20,21]. In contrast to techniques based on near-resonant laser beams, heating of the atomic cloud due to spontaneous emission is negligible [22]. The position dependence of the on-site modulation introduced by the running-wave beams leads to a spatially dependent complex tunneling amplitude. Therefore, an atom hopping around a closed loop acquires a nontrivial phase, which mimics an Aharonov-Bohm phase. In our setup, we realize a uniform effective flux of $\Phi=\pi / 2$ per plaquette, whose value is fully tunable. We study resonant laser-assisted tunneling in the tilted optical potential and reveal the local distribution of fluxes by partitioning the lattice into isolated four-site square plaquettes. Furthermore, we show that for two spin states with opposite magnetic moments $|\uparrow\rangle$ and $|\downarrow\rangle$, our coupling scheme directly gives rise to a non-Abelian $\mathrm{SU}(2)$ gauge field that results in opposite magnetic fields for $|\uparrow\rangle$ and $|\downarrow\rangle$ particles. In the presence of such a gauge field, the tightbinding Hamiltonian is time-reversal symmetric and corresponds precisely to the one underlying the quantum spin Hall effect [7,23]. 
Our experimental setup consists of an ultracold gas of ${ }^{87} \mathrm{Rb}$ atoms held in a three-dimensional optical lattice created by three mutually orthogonal standing waves of laser light at wavelengths $\lambda_{x}=\lambda_{y}=767 \mathrm{~nm}$ and $\lambda_{z}=$ $844 \mathrm{~nm}$. The depth of the lattice along $z$ is chosen deep enough to suppress tunneling between individual planes, typically $V_{z}=30(1) E_{r z}$, where $E_{r i}=h^{2} /\left(2 m \lambda_{i}^{2}\right), \quad i \in$ $\{x, y, z\}$, are the corresponding recoil energies and $m$ is the mass of an atom. A magnetic field gradient $B^{\prime}$ along $x$ is used to generate a linear potential of amplitude $\pm \Delta$ between neighboring sites, depending on the internal state of the atom (Fig. 1). We use two Zeeman states with opposite magnetic moments denoted as spin-up $|\uparrow\rangle \equiv$ $\left|F=1, m_{F}=-1\right\rangle$ and spin-down $|\downarrow\rangle \equiv\left|F=2, m_{F}=-1\right\rangle$. For $\Delta \gg J_{x}$, with $J_{x}$ being the bare coupling along $x$, tunneling is inhibited and can be restored resonantly using a pair of far-detuned running-wave beams with a frequency difference $\omega=\Delta / \hbar$ (Fig. 1). The local optical potential created by these two beams is $V_{K}(\mathbf{r})=$ $V_{K}^{0} \cos ^{2}(\mathbf{q} \cdot \mathbf{r} / 2+\omega t / 2)$, with $\mathbf{q}=\mathbf{k}_{\mathbf{1}}-\mathbf{k}_{\mathbf{2}}$ being the wave vector difference. This gives rise to a time-dependent on-site modulation term with spatially dependent phases $\phi_{m, n}=\mathbf{q} \cdot \mathbf{R}$, where $\mathbf{R}=m \mathbf{d}_{\mathbf{x}}+n \mathbf{d}_{\mathbf{y}}$ denotes the lattice site $(m, n)$. In the high-frequency limit $\hbar \omega \gg J_{i}, i \in\{x, y\}$, the system can be described by an effective timeindependent Hamiltonian

$$
\hat{H}_{\uparrow, \downarrow}=-\sum_{m, n}\left(K \mathrm{e}^{ \pm i \phi_{m, n}} \hat{a}_{m+1, n}^{\dagger} \hat{a}_{m, n}+J \hat{a}_{m, n+1}^{\dagger} \hat{a}_{m, n}\right)+\text { H.c., }
$$
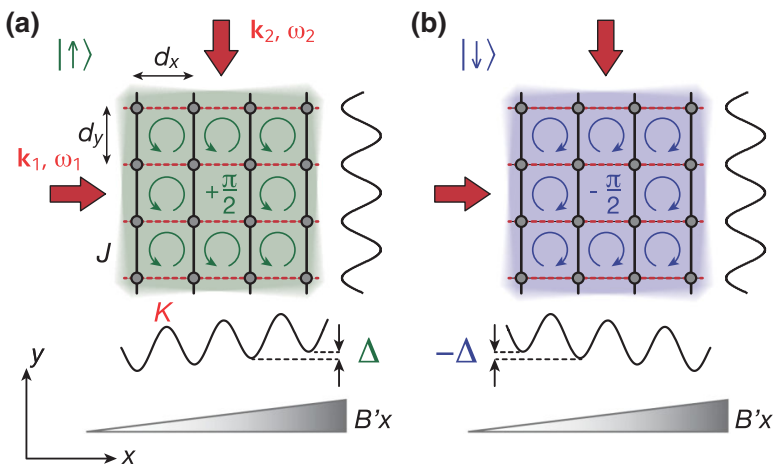

FIG. 1 (color). Experimental setup. The experiment consists of a 3D optical lattice, where the vertical lattice isolates different planes. The lattice constants within each plane are $\left|\mathbf{d}_{i}\right|=\lambda_{i} / 2$, with $i=x, y$. Along $y$, bare tunneling occurs with strength $J$, while tunneling along $x$ is inhibited by a magnetic field gradient $B^{\prime}$, which introduces an energy offset between neighboring sites of (a) $\Delta$ for $|\uparrow\rangle$ atoms and (b) $-\Delta$ for $|\downarrow\rangle$ atoms. An additional pair of laser beams (red arrows) with wave vectors $\left|\mathbf{k}_{1}\right| \simeq\left|\mathbf{k}_{2}\right|=$ $2 \pi / \lambda_{K}$ and frequency difference $\omega=\omega_{1}-\omega_{2}=\Delta / \hbar$ is used to restore resonant tunneling with complex amplitude $K$. This realizes an effective flux of (a) $\Phi=\pi / 2$ for $|\uparrow\rangle$ atoms and (b) $-\Phi$ for $|\downarrow\rangle$ atoms. where the sign of the phase factor is positive for $|\uparrow\rangle$ atoms and negative for $|\downarrow\rangle$ atoms. In the limit of $\Delta \gg V_{K}^{0}$, the effective coupling strengths along $x$ and $y$ are $K=J_{x} \mathcal{J}_{1}\left(V_{K}^{0} /(\sqrt{2} \Delta)\right) \simeq J_{x} V_{K}^{0} /(2 \sqrt{2} \Delta)$ and $J=$ $J_{y} \mathcal{J}_{0}\left(V_{K}^{0} /(\sqrt{2} \Delta)\right) \simeq J_{y}$, where $\mathcal{J}_{\nu}(x)$ are the Bessel functions of the first kind [24]. We note that the spin-dependent Peierls phase factors directly arise from the spin-dependent Zeeman coupling to the real applied magnetic field gradient.

For the chosen propagation of the running-wave beams shown in Fig. 1 and $\lambda_{K}=2 \lambda_{y}$, we obtain a phase factor $\phi_{m, n}=\pi / 2(m+n)$ [22]. Therefore, the phase accumulated on a closed path around a plaquette is $\pm \Phi= \pm \pi / 2$, depending on the spin of the particle, and the corresponding gauge field is given by $\mathcal{A}=-(\hbar \Phi(x+y) /$ $\left.\left(d_{x} d_{y}\right), 0,0\right) \hat{\sigma}_{z}$, where $\hat{\sigma}_{z}$ is the Pauli $z$ matrix. A different value of the flux $\Phi$ could be achieved by changing the wavelength $\lambda_{K}$ or the angle between the running-wave beams.

To study laser-assisted tunneling in the presence of the magnetic field gradient $B^{\prime}$, we loaded a Bose-Einstein condensate of about $5 \times 10^{4}$ atoms in an initial state, where all atoms populated even sites with at most one atom per site, while odd sites were left empty \{Fig. 2(a) and Ref. [22] $\}$. The final lattice depths $V_{x}=5.0(1) E_{r x}$ and $V_{y}=40(1) E_{r y}$ were chosen to yield a negligible tunneling along $y$ and a bare tunnel coupling along $x$ of $J_{x} / h=$ $0.26(1) \mathrm{kHz}$. Because of the magnetic field gradient, tunneling was inhibited along $x$ and all atoms stayed in even sites. The running-wave beams were then switched (a)

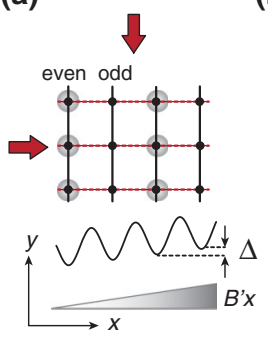

(b)

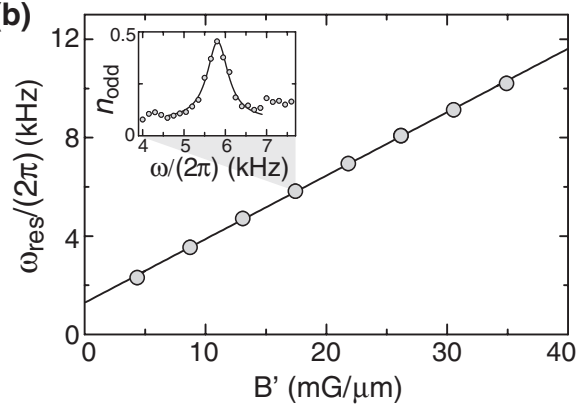

FIG. 2 (color). Laser-assisted tunneling in a tilted optical lattice. (a) Schematic of the initial state used to study laserassisted tunneling in the presence of a magnetic field gradient $B^{\prime}$. Atoms are initially prepared in even sites with at most one atom per lattice site, while odd sites are left empty. (b) Measured frequency difference $\omega_{\text {res }}=\omega_{1}-\omega_{2}$, where atoms resonantly tunnel from even to odd sites, as a function of the magnetic field gradient $B^{\prime}$. The finite value at $B^{\prime}=0$ is due to a small additional magnetic field gradient [22]. The solid line is a linear fit to our data. The inset shows a typical spectroscopy measurement for $B^{\prime}=17.5 \mathrm{mG} / \mu \mathrm{m}$, where the fraction of atoms on odd sites $n_{\text {odd }}$ is measured as a function of the frequency difference $\omega$ to determine the resonance frequency $\omega_{\text {res }}$. The solid line shows the fit of a Lorentzian function to our data. 
on for $4 \mathrm{~ms}$ with strength $V_{K}^{0}=9.9(2) E_{r K}$, where $E_{r K}=$ $h^{2} /\left(2 m \lambda_{K}^{2}\right)$. Afterwards, we measured the fraction of atoms transferred to odd sites $n_{\text {odd }}$ as a function of the frequency difference $\omega$ for a fixed value of the magnetic field gradient. Even-odd resolved detection was achieved by transferring atoms in odd sites to a higher Bloch band and applying a subsequent band-mapping sequence $[22,25]$. As shown in the inset of Fig. 2(b), atoms are transferred resonantly to odd sites when the frequency of the running-wave beams matches the energy offset $\Delta$ between neighboring sites. We measured the resonance frequency $\omega_{\text {res }}$ for various values of the magnetic field gradient and observed a large tunability up to about $\Delta / h \sim 10 \mathrm{kHz}$ [Fig. 2(b)].

The spatial distribution of the local fluxes induced by the running-wave beams was revealed by a series of measurements in isolated four-site square plaquettes using optical superlattices. This was achieved by superimposing two additional standing waves along $x$ and $y$ with wavelength $\lambda_{l i}=2 \lambda_{i}, i \in\{x, y\}$. The resulting potential along $x$ is $V(x)=V_{l x} \sin ^{2}\left(k_{x} x / 2+\varphi_{x} / 2\right)+V_{x} \sin ^{2}\left(k_{x} x\right)$, where $V_{l x}$ is the depth of the "long" lattice. The superlattice potential along $y$ is given by an analogous expression. The depths of the lattices and the relative phases $\varphi_{x}$ and $\varphi_{y}$ can be controlled independently. For $\varphi_{x}=\varphi_{y}=0$, we realize symmetric double well potentials along $x$ and $y$ to isolate individual plaquettes (Fig. 3). Because of the presence of the magnetic field gradient, the plaquettes are tilted along $x$, with an energy offset $\Delta$ for $|\uparrow\rangle$ atoms and $-\Delta$ for $|\downarrow\rangle$ atoms. The four sites of the plaquette are denoted as $A, B$, $C$, and $D$ (Fig. 3). The experiment started by loading spin-polarized single atoms into the ground state of the tilted plaquettes: $\left|\Psi_{\uparrow}^{0}\right\rangle=(|A\rangle+|D\rangle) / \sqrt{2}$ and $\left|\Psi_{\downarrow}^{0}\right\rangle=$ $(|B\rangle+|C\rangle) / \sqrt{2}$, for $|\uparrow\rangle$ and $|\downarrow\rangle$, respectively \{Fig. 3(a) and Ref. [22]\}. After switching on the running-wave beams, the atoms couple to the $B$ and $C$ sites ( $|\uparrow\rangle$ atoms) and $A$ and $D$ sites ( $|\downarrow\rangle$ atoms). Without the artificial magnetic field, the atoms would oscillate periodically between left and right, but due to the phase imprinted by the running-wave beams, the atoms experience a force perpendicular to their velocity similar to the Lorentz force acting on a charged particle in a magnetic field. We measured the time evolution of the atom population on different bonds $\left(N_{\text {left }}=N_{A}+N_{D}, \quad N_{\text {right }}=N_{B}+N_{C}, \quad N_{\text {up }}=N_{C}+N_{D}\right.$, and $\left.N_{\text {down }}=N_{A}+N_{B}\right)$, with $N_{q}$ being the atom population per site $(q=A, B, C, D)$, by applying the even-odd resolved detection along both directions independently [22]. From this, we obtained the mean atom positions along $x$ and $y,\langle X\rangle=\left(N_{\text {right }}-N_{\text {left }}\right) d_{x} / 2 N$ and $\langle Y\rangle=$ $\left(N_{\text {up }}-N_{\text {down }}\right) d_{y} / 2 N$, with $N$ being the total atom number. As shown in Fig. 3(a), the mean atom position follows a small-scale quantum analog of the classical cyclotron orbit for charged particles. Starting with equally populated sites $A$ and $D$, spin-up atoms experience a force along $y$, which is perpendicular to the initial velocity and points towards
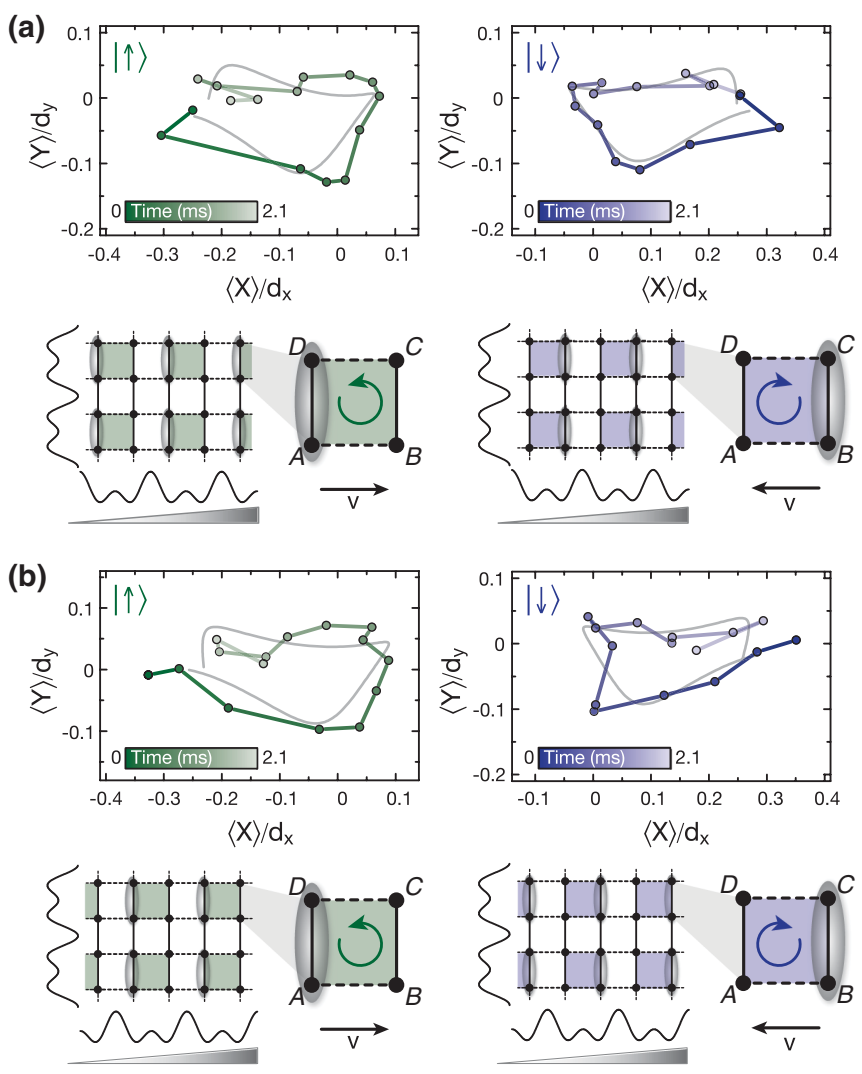

FIG. 3 (color). Quantum cyclotron orbits obtained from the mean atom positions along $x$ and $y,\langle X\rangle / d_{x}$ and $\langle Y\rangle / d_{y}$, for $J / K \approx 2$ [22]. Every data point is an average over three individual measurements. The solid gray lines show the fit of the theoretically expected evolution to the data, which was obtained from a numerical calculation solving the time-dependent Schrödinger equation of the $4 \times 4$ Hamiltonian. The oscillation amplitudes and offsets were fitted independently along $x$ and $y$, whereas the time offset $\tau=0.12(5) \mathrm{ms}$ and flux $\Phi=0.73(5) \times$ $\pi / 2$ were fixed (see the main text and the Supplemental Material [22]). The schematics illustrate the superlattice potentials used to partition the lattice into plaquettes together with the initial state for $|\uparrow\rangle$ atoms (green) and $|\downarrow\rangle$ atoms (blue) and the direction of the flux. The superlattice potential along $x$ is shifted by one lattice constant for the experimental results in (b) with respect to the ones in (a) to demonstrate the uniformity of the artificial magnetic field.

the lower bond in the plaquette ( $A$ and $B$ sites). Spin-down atoms, initially with opposite velocity, also move towards the lower bond. Therefore, the chirality of the cyclotron orbit is reversed, revealing the spin-dependent nature of the artificial magnetic field [Fig. 3(a)]. The value of the magnetic flux per plaquette $\Phi=0.73(5) \times \pi / 2$, measured in our previous work [12], is used for the fits in Fig. 3. The difference from $\Phi=\pi / 2$, expected for a homogeneous lattice, stems from the smaller distance between lattice sites inside the plaquettes when separated.

To further demonstrate the uniformity of the magnetic field, we performed the same set of measurements in plaquettes shifted by one lattice constant along $x$. This 
(a)

(b)
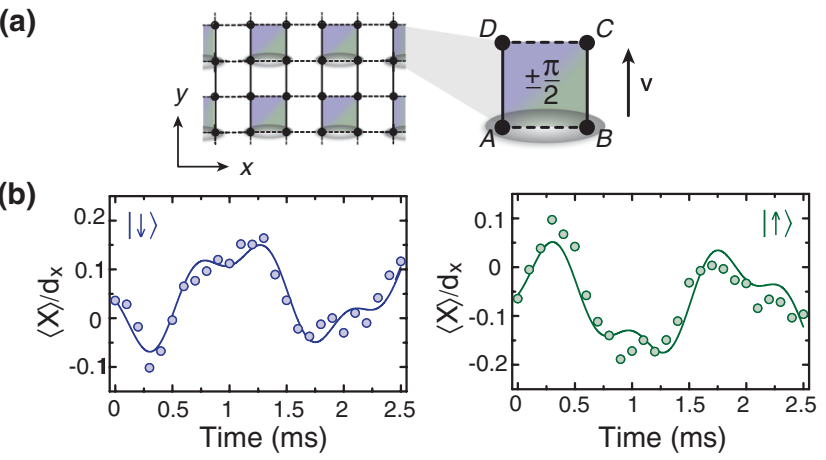

(c)

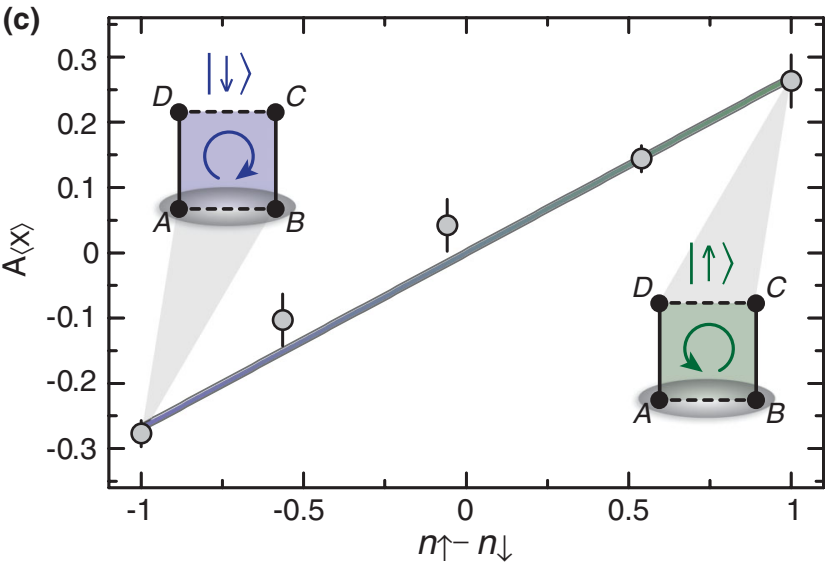

FIG. 4 (color). Particle currents as a function of the spin imbalance of the system. (a) Schematic of the initial-state configuration with one atom in a spin-mixture state of $|\downarrow\rangle$ and $|\uparrow\rangle$ in the lower bond of each plaquette [22]. (b) Evolution of the mean atom position along $x$ for all particles in $|\downarrow\rangle$ (blue) and all atoms in $|\uparrow\rangle$ (green) for $J / h=0.69(1) \mathrm{kHz}, K / h=$ $0.38(1) \mathrm{kHz}$, and $\Delta / h=5.31(5) \mathrm{kHz}$. The solid lines show a fit of the theoretically expected curve to the data obtained using the same method as in Fig. 3 with a time offset $\tau=0.18(3) \mathrm{ms}$. (c) Oscillation amplitude $A_{\langle X\rangle}=A_{\langle X\rangle}^{\downarrow}+A_{\langle X\rangle}^{\uparrow}$ of the mean atom position $\langle X\rangle / d_{x}$ as a function of the spin imbalance $n_{\uparrow}-n_{\downarrow}$. Every data point is an average over two individual measurements. The insets show schematics of the initial states together with the directions of the flux for all atoms in $|\downarrow\rangle$ (blue) and $|\uparrow\rangle$ (green). The solid line is a linear fit to our data, with the offset set to zero. The error bars show the standard deviation of our data.

was achieved by changing the relative phase between the two standing waves along $x$ from $\varphi_{x}=0$ [Fig. 3(a)] to $\varphi_{x}=\pi$ [Fig. 3(b)]. The chirality of the obtained cyclotron orbits remained unchanged, which implies that a homogenous magnetic flux is present in the system.

In analogy to the spin Hall effect observed in solid-state devices [26], we measured the particle current perpendicular to the initial motion as a function of spin imbalance $n_{\uparrow}-n_{\downarrow}$, where $n_{\uparrow}\left(n_{\downarrow}\right)$ is the fraction of spin-up (down) atoms. The experimental sequence started from a Mott insulator of unit filling, with each atom prepared in a superposition of $|\uparrow\rangle$ and $|\downarrow\rangle$. We then loaded single atoms into the ground state of the lower bond of the plaquettes, which has an equal weight on $A$ and $B$ sites [Fig. 4(a)], and measured the mean atom position $\langle X\rangle / d_{x}$. We obtained an oscillation amplitude for a spin-polarized state of $A_{\langle X\rangle}=$ $-0.28(2)$ for $|\downarrow\rangle$ atoms and $A_{\langle X\rangle}=0.26(4)$ for $|\uparrow\rangle$ atoms. As can be seen in Fig. 4(b), the evolution is almost perfectly mirrored for the two spin components. The measured oscillation amplitude as a function of the spin imbalance $n_{\uparrow}-n_{\downarrow}$ shows that the current depends linearly on the spin imbalance and reverses sign when flipping the spin [Fig. 4(c)].

In conclusion, we have demonstrated a new type of optical lattice that realizes the non-time-reversalsymmetric Hofstadter-Harper Hamiltonian and the timereversal-symmetric quantum spin Hall Hamiltonian for ultracold atoms in optical lattices. Loading spin-polarized or two-component Fermi gases into this lattice should allow one to directly realize quantum Hall and $Z_{2}$ topological insulators with chiral and helical edge states for finite sized systems. This system also opens the path to explore the fractal band structure of the Hofstadter butterfly with ultracold atoms [6]. The lowest band is topologically equivalent to the lowest Landau level and exhibits a Chern number of one $[11,17,19,27]$. In future experiments, the ground-state properties, the effect of the Berry curvature, and topological edge states could be studied [28-30]. The chiral edge modes in this lattice could be directly revealed in ladder systems exposed to a homogeneous magnetic field, which constitute the smallest possible 2D systems in which these states can be observed [31]. Moreover, our work constitutes an important step towards the study of the quantum Hall effect with ultracold atomic gases and the creation of strongly interacting fractional quantum-Hall-like liquids for bosonic and fermionic atoms [32].

We thank S. Nascimbène and Y.-A. Chen for their help in setting up the experiment and sharing of their ideas. This work was supported by the DFG (FOR635, FOR801), DARPA (OLE programm), and NIM. M. Aidelsburger was additionally supported by the Deutsche Telekom Stiftung.

Note added.-Recently, we became aware of similar work carried out by Ketterle and co-workers [33].

[1] D. Jaksch and P. Zoller, Ann. Phys. (Amsterdam) 315, 52 (2005); I. Bloch, J. Dalibard, and W. Zwerger, Rev. Mod. Phys. 80, 885 (2008).

[2] M. Lewenstein, A. Sanpera, V. Ahufinger, B. Damski, A. Sen(De), and U. Sen, Adv. Phys. 56, 243 (2007); N. Cooper, Adv. Phys. 57, 539 (2008); A. Fetter, Rev. Mod. Phys. 81, 647 (2009).

[3] I. Bloch, J. Dalibard, and S. Nascimbène, Nat. Phys. 8, 267 (2012).

[4] W. S. Bakr, A. Peng, M.E. Tai, R. Ma, J. Simon, J. I. Gillen, S. Fölling, L. Pollet, and M. Greiner, Science 329, 547 (2010); J. F. Sherson, C. Weitenberg, M. Endres, M. Cheneau, I. Bloch, and S. Kuhr, Nature (London) 467, 68 
(2010); C. Weitenberg, M. Endres, J.F. Sherson, M. Cheneau, P. Schauß, T. Fukuhara, I. Bloch, and S. Kuhr, Nature (London) 471, 319 (2011).

[5] P. Harper, Proc. Phys. Soc. London Sect. A 68, 874 (1955).

[6] D. Hofstadter, Phys. Rev. B 14, 2239 (1976).

[7] B. A. Bernevig and S.-C. Zhang, Phys. Rev. Lett. 96, 106802 (2006).

[8] K. W. Madison, F. Chevy, W. Wohlleben, and J. Dalibard, Phys. Rev. Lett. 84, 806 (2000); J. Abo-Shaeer, C. Raman, J. Vogels, and W. Ketterle, Science 292, 476 (2001).

[9] V. Schweikhard, I. Coddington, P. Engels, V.P. Mogendorff, and E. A. Cornell, Phys. Rev. Lett. 92, 040404 (2004); V. Bretin, S. Stock, Y. Seurin, and J. Dalibard, ibid. 92, 050403 (2004).

[10] Y. Lin, R. L. Compton, K. Jiménez-García, J. V. Porto, and I. B. Spielman, Nature (London) 462, 628 (2009).

[11] J. Dalibard, F. Gerbier, G. Juzeliūnas, and P. Öhberg, Rev. Mod. Phys. 83, 1523 (2011).

[12] M. Aidelsburger, M. Atala, S. Nascimbène, S. Trotzky, Y.-A. Chen, and I. Bloch, Phys. Rev. Lett. 107, 255301 (2011); Appl. Phys. B, doi: 10.1007/s00340-013-5418-1 (2013).

[13] J. Struck, C. Ölschläger, R. Le Targat, P. Soltan-Panahi, A. Eckardt, M. Lewenstein, P. Windpassinger, and $\mathrm{K}$. Sengstock, Science 333, 996 (2011).

[14] K. Jiménez-García, L. J. LeBlanc, R. A. Williams, M. C. Beeler, A. R. Perry, and I. B. Spielman, Phys. Rev. Lett. 108, 225303 (2012).

[15] J. Struck, C. Ölschläger, M. Weinberg, P. Hauke, J. Simonet, A. Eckardt, M. Lewenstein, K. Sengstock, and P. Windpassinger, Phys. Rev. Lett. 108, 225304 (2012).

[16] M. C. Beeler, R. A. Williams, K. Jiménez-García, L. J. LeBlanc, A. R. Perry, and I. B. Spielman, Nature (London) 498, 201 (2013).

[17] D. Jaksch and P. Zoller, New J. Phys. 5, 56 (2003).

[18] F. Gerbier and J. Dalibard, New J. Phys. 12, 033007 (2010).

[19] E. J. Mueller, Phys. Rev. A 70, 041603 (2004).

[20] A. Kolovsky, Europhys. Lett. 93, 20003 (2011); C. E. Creffield and F. Sols, Europhys. Lett. 101, 40001 (2013).
[21] A. Bermudez, T. Schaetz, and D. Porras, Phys. Rev. Lett. 107, 150501 (2011).

[22] See Supplemental Material at http://link.aps.org/ supplemental/10.1103/PhysRevLett.111.185301 for the estimated heating rate, local phase distribution, experimental sequence, detection methods, and numerical simulations.

[23] N. Goldman, I. Satija, P. Nikolic, A. Bermudez, M. A. Martin-Delgado, M. Lewenstein, and I. B. Spielman, Phys. Rev. Lett. 105, 255302 (2010).

[24] F. Grossmann and P. Hänggi, Europhys. Lett. 18, 571 (1992); M. Holthaus, Phys. Rev. Lett. 69, 351 (1992).

[25] J. Sebby-Strabley, M. Anderlini, P.S. Jessen, and J. V. Porto, Phys. Rev. A 73, 033605 (2006); S. Fölling, S. Trotzky, P. Cheinet, M. Feld, R. Saers, A. Widera, T. Müller, and I. Bloch, Nature (London) 448, 1029 (2007).

[26] Y. K. Kato, R. C. Myers, A.C. Gossard, and D. D. Awschalom, Science 306, 1910 (2004); J. Wunderlich, B. Kaestner, J. Sinova, and T. Jungwirth, Phys. Rev. Lett. 94, 047204 (2005).

[27] N. R. Cooper, Phys. Rev. Lett. 106, 175301 (2011); N. R. Cooper and J. Dalibard, Europhys. Lett. 95, 66004 (2011).

[28] S. T. Baur and N.R. Cooper, Phys. Rev. A 88, 033603 (2013).

[29] H. M. Price and N. R. Cooper, Phys. Rev. A 85, 033620 (2012).

[30] N. Goldman, J. Dalibard, A. Dauphin, F. Gerbier, M. Lewenstein, P. Zoller, and I. B. Spielman, Proc. Natl. Acad. Sci. U.S.A. 110, 6736 (2013).

[31] D. Hügel and B. Paredes, arXiv:1306.1190.

[32] A. S. Sørensen, E. Demler, and M. D. Lukin, Phys. Rev. Lett. 94, 086803 (2005); R. N. Palmer and D. Jaksch, ibid. 96, 180407 (2006); M. Hafezi, A. S. Sørensen, E. Demler, and M. D. Lukin, Phys. Rev. A 76, 023613 (2007); G. Möller and N. R. Cooper, Phys. Rev. Lett. 103, 105303 (2009).

[33] H. Miyake, G. A. Siviloglou, C. J. Kennedy, W. C. Burton, and W. Ketterle, following Letter, Phys. Rev. Lett. 111, 185302 (2013). 